\title{
Rechtsinformationen aus Datenbanken
}

\author{
Die Möglichkeiten und Kosten der Online-Anbindung \\ Rüdiger Mähler*
}

\section{Rechtsinformationen aus Datenbanken}

Das wachsende Interesse der Anwaltschaft am Einsatz neuer Technologien für die Aufgabenerledigung ist nicht zu verkennen. Der Nutzen, insbesondere elektronischer Datenverarbeitungs- und Kommunikationstechniken als Organisations- und Rationalisierungsinstrument für die Anwaltskanzleien, wird auch mit dem Ziel einer Verbesserung der Dienstleistung von einer immer größer werdenden Anzahl Rechtsanwälte erkannt.

Welche Aufgabenfelder durch den Einsatz dieser Technologien effizienter bearbeitet werden können und welche organisatorischen Schwachstellen im Kanzleibetrieb zu beseitigen sind, wurde nach einer intensiven empirischen Studie, in der Aufsatzreihe "Wege zur modernen Bürokommunikation" (Informatik u. Recht 11-12/87, 01/88) beschrieben.

Neben den Aspekten der Organisation und der Rationalisierung darf der Rechtsanwalt seine eigene Position als Jurist und Unternehmer im Dienstleistungswettbewerb nicht unberücksichtigt lassen. Mittel- und langfristig können nur die Rechtsanwaltskanzleien wirtschaftlich gesund bleiben, die sowohl organisatorisch als auch in der Qualität ihrer juristischen Sachbearbeitung überzeugen können.

Die Tatsache, daß der Anwalt praktisch verpflichtet ist, die wesentliche Rechtsprechung aus den von ihm bearbeiteten Rechtsgebieten zu kennen und der ständig zunehmende Umfang an Gesetzen und Entscheidungen, erfordert den Einsatz neuer Informationstechnologien.

Der Rechtsanwalt wird zukünftig immer weniger in der Lage sein, die Vielfalt der notwendigen Informationen ohne diese technische Unterstützung sicher und aktuell zu beherrschen.

Daß dieser Effekt bereits heute deutlich wird, konnte durch empirische Studien und zahlreiche Gespräche mit Praktikern (Rechtsanwalt im Kanzleialltag) festgestellt werden.

Bei Zusammenfassung der wesentlichen Punkte wurde fast einheitlich die Situation wie folgt geschildert:

In einigen Fällen $(3-5 \%$ der zu bearbeitenden Rechtsfälle) reichen dem Rechtsanwalt weder seine im Gedächtnis vorhandenen Rechtskenntnisse aus, noch kann er die fehlenden Informationen ohne besondere Mühe aus seiner Bibliothek entnehmen. Hier werden umfangreiche Recherchen in Bibliotheken erforderlich. Der Zeitaufwand für die Bearbeitung ist sehr groß.
Die genannten Fälle sind diejenigen, die auf dem Schreibtisch liegenbleiben und fast immer erst in letzter Minute erledigt werden. Kurz gesagt, es sind diejenigen Fälle, die den eigentlichen Streß verursachen, weil der Anwalt keine Ansatzpunkte findet.

Bei den Informationen, auf die es hier ankommt, handelt es sich sowohl um besonders aktuelle (Datenbank für den Informationszuwachs), als auch um besonders spezielle Informationen (Spezialdatenbank).

\section{Der Bedarf des Anwaltes}

Betrachtet man den hieraus abgeleiteten Bedarf des Anwaltes als Praktiker, so ist festzuhalten, daß es sich hier um eine sporadische Nutzung von Datenbanken handelt. Der Praktiker hat zunächst großen Bedarf an unmittelbaren und themenspezifischen Hilfestellungen für seinen Rechtsalltag.

Dieser praktische Bedarf ließe sich zenächst durch relativ einfache Datenbanken decken. Der Einstieg für einen aktuellen Informationsanbieter wäre zunächst darin zu sehen, daß er die juristischen Informationen, die seit der jeweils letzten Ausgabe des „Palandt“ zugewachsen sind, über eine Datenbank annietet (nach Möglichkeit gleiche Suchstruktur wie beim Palandt) bzw. daß die Leitsatzdatei der NJW als Datenbank zur Verfügung gestellt wird.

Ein Anbieter für Spezialdatenbanken hat bestimmt Chancen am Markt, wenn er fundierte Hilfestellungen für den Praktiker innerhalb bestinmmter Aufgabeníelder (Sachgebiete) bietet.

Datenbanken müssen auf lange Sicht die Funktion einer umfassenden und aktuellen Informationsquelle für den Rechisanwalt ühernelnnen.

\section{Entscheidungskriterien}

Wesentliche Entscheidungskriterien für die Akzeptanz einer Datenbank sind:

- Umfassender und aktueller Informationsgehalt

- Schneller und einfacher Suchvorgang

- Hoher Anteil an brauchbaren Entseneidungen (wenig Fehlversuche)

- Einfache Verwertbarkeit der Information

- Kurze Zugriffszeiten

* Dipl. Ing. Rüdiger Mähler ist Mitglied der Projektgruppe „Moderne Bürokommunikation“ der Deutschen Bundespost beim Fernmeldeamt Hagen 
Neben diesen Entscheidungskriterien sind insbesondere bei sporadischen Nutzern von Datenbanken die Kosten für die vorzuhaltenden technischen Einrichtungen (Hardware und Software) und die Übertragungskosten ein wichtiger Entscheidungsgrund.

\section{Online Anbindung}

Für die online Übertragung der Datenbankinhalte gibt es mehrere unterschiedliche Möglichkeiten. Die Entscheidung für die richtige Online-Anbindung ist für den potentiellen Nutzer nieht leicht. Um hier eine Hilfestellung sowohl dem Nutzer als auch den Datenbankanbietern zu geben ist im folgenden ein Anwendungsbeispiel aufgeführt und durchgerechnet worden.

\section{Vergleichsbetrachtung der Übertragungskosten}

In Ihrer Rechtsanwaltskanzlei soll der Zugang zu Datenbanken realisiert werden. Es wird ein zentraler Anschluß in der Kanzlei unterstellt, der im „online-Betrieb“ mit dem Rechenzentrum zusammenarbeitet.

\section{Vorgaben für den Gebührenvergleich:}

Übertragungsgeschwindigkeit $2400 \mathrm{bit} / \mathrm{sec}$

Entfernung $105 \mathrm{~km}$

Anzahl Transaktionen/Tag 6

Dauer einer Transaktion $240 \mathrm{sec}$

Anzahl Zeichen bei der

Rückantwort des Rechners

Arbeitstage/Monat

Dialogübertragung

ca. 2000 Zeichen
20

einfache Datenabfrage

Es sind keine Kosten für die jeweilige Datenbankinformation enthalten.

Dies ist ein reiner Vergleich der Kosten für die Kommunikation. In den Vergleich sind nicht eingeflossen:

a) die Endgerätekosten,

b) die unterschiedlichen Bedienprozeduren mit ihren organisatorischen Vor- oder Nachteilen,

c) die Kommunikationssicherheit.

Realisierung im öffentlichen Fernsprechnetz:

\section{Monatliche Gebübren:}

1.11 Fernsprechhauptanschluß

1.21 Modem 1200-01

+ Instandhaltung

+ Meß- und Prüfaufwand

\section{Verkebrsgebübren $(V g)$ :}

Entfernung - $100 \mathrm{~km}$, daher $0,23 \mathrm{DM}$ je $12 \mathrm{sec}$ Übertragungszeit

$\mathrm{Vg}=\frac{240 \mathrm{sec}}{12} \times 6 \times 20 \times 0,23 \mathrm{DM}$

$\mathrm{Vg}=552,-\mathrm{DM}$

$3,00 \mathrm{DM}$

$5,00 \mathrm{DM}$

$42,75 \mathrm{DM}$
3. Monatliche Gesamtgebübren:

43,75 DM

$+552,00 \mathrm{DM}$

$595,75 \mathrm{DM}$

Realisierung mit Alexis

Monatliche Gebübren:

Grundgebühr für eine Box $50,-$ DM

Telefonanschluß 27,-DM

Modem D 1200 S 30,- DM

Wartung 15,- DM

Meß- und Prüfaufwand 5,- DM

Verkebrsgebübren ( $V g)$ :

Verbindungsgebühr Tele-

fonnetz (8 Minuten-Takt

unterstellt)

$6 \times 20 \times 0,23$

Teilnehmerkennung (NUI)

Zugangsgebühr

$(0,05 \mathrm{DM} / \mathrm{Min} \times 6 \times 4 \times 20) \quad 24,-\mathrm{DM}$

PAD-Gebühr

$(0,06 \mathrm{DM} / \mathrm{Min} \times 6 \times 4 \times 20) \quad 28,80 \mathrm{DM}$

Zuschlag für Verbindungsgebühr

$(0,05 \mathrm{DM} \times 20)$

Zeitgebühr bei GVV

$(0,01 \times 6 \times 4 \times 20)$

$27,60 \mathrm{DM} * 138,-\mathrm{DM}$

$15,-\mathrm{DM}$

6,- DM

4,80 DM

Volumengebühr

$\left(\frac{2000}{64} \times 1,2 \times 6 \times 20 \times\right.$

0,033 DM)

$14,85 \mathrm{DM}$

Belegungsgebühr TELE-

BOX

$(6 \times 4 \times 20 \times 0,25 \mathrm{DM}) \quad 120,-\mathrm{DM}$

Adressiergebühr

$(6 \times 20 \times 0,07 \mathrm{DM})$

$\frac{8,40 \mathrm{DM}}{76,45 \mathrm{DM}} \overline{486,85 \mathrm{DM}}$

* bei künftig 0,23 DM je $50 \mathrm{sec}$

Übertragungszeit $5 \times 6 \times 20 \times 0,23 \mathrm{DM}$

\section{Realisierung mit Deutscher Mailbox}

\section{Monatliche Gebühren:}

Grundgebühr für eine Box

Telefonanschluß

40,- DM

Modem D $1200 \mathrm{~S}$

Wartung

Meß- und Prüfaufwand

$27,-\mathrm{DM}$

$30,-\mathrm{DM}$

$15,-\mathrm{DM}$

$5,-\mathrm{DM}$

Verkehrsgebühren ( $V g$ ):

Verbindungsgebühr Tele-

fonnetz (8 Minuten-Takt

unterstellt)

$6 \times 20 \times 0,23$

$27,60 \mathrm{DM}^{*} 138,-\mathrm{DM}$ 


\begin{tabular}{|c|c|c|}
\hline $\begin{array}{l}\text { Teilnehmerkennung (NUI) } \\
\text { Zugangsgebühr }\end{array}$ & $15,-\mathrm{DM}$ & \\
\hline$(0,05 \mathrm{DM} / \mathrm{Min} \times 6 \times 4 \times 20)$ & $24,-\mathrm{DM}$ & \\
\hline $\begin{array}{l}\text { PAD-Gebühr } \\
(0,06 \mathrm{DM} / \mathrm{Min} \times 6 \times 4 \times 20) \\
\text { Zuschlag für Verbindungs- } \\
\text { gebühr }\end{array}$ & $28,80 \mathrm{DM}$ & \\
\hline $\begin{array}{l}(0,05 \mathrm{DM} \times 20) \\
\text { Zeitgebühr bei } \mathrm{GVV}\end{array}$ & $6,-\mathrm{DM}$ & \\
\hline $\begin{array}{l}(0,01 \times 6 \times 4 \times 20) \\
\text { Volumengebühr } \\
\left(\frac{2000}{64} \times 1,2 \times 6 \times 20 \times\right.\end{array}$ & $4,80 \mathrm{DM}$ & \\
\hline $\begin{array}{l}\text { 0,033 DM) } \\
\text { Belegungsgebühr TELE- } \\
\text { BOX }\end{array}$ & $14,85 \mathrm{DM}$ & \\
\hline $\begin{array}{l}(6 \times 4 \times 20 \times 0,25 \mathrm{DM}) \\
\text { Adressiergebühr }\end{array}$ & $120,-\mathrm{DM}$ & \\
\hline$(6 \times 20 \times 0,07 \mathrm{DM})$ & $\frac{8,40 \mathrm{DM}}{369,45 \mathrm{DM}}$ & $\overline{476,85 \mathrm{DM}}$ \\
\hline
\end{tabular}

* bei künftig $0,23 \mathrm{DM}$ je $50 \mathrm{sec}$

Übertragungszeit $5 \times 6 \times 20 \times 0,23 \mathrm{DM}$

\section{Realisierung mit TELEBOX}

(Voraussetzung: Übergang zu Datenbanken aus dem TELEBOX-Dienst ist realisiert (Gateway-Funktion))

Für den TELEBOX-Dienst wird Zugang aus dem FeNetz, als Datex-P 20 F Hauptanschluß $1200 \mathrm{bit} / \mathrm{sec}$ mit Modem D 1200 S unterstellt.

Monatliche Gebübren:

Grundgebühr für eine Box

Telefonanschluß

Modem D $1200 \mathrm{~S}$

Wartung

Meß- und Prüfaufwand

Verkebrsgebübren $(V g)$ :

Verbindungsgebühr Tele-

fonnetz 8 Minuten-Takt

unterstellt)

$6 \times 20 \times 0,23$

Teilnehmerkennung (NUI)

Zugangsgebühr

$(0,05 \mathrm{DM} / \mathrm{Min} \times 6 \times 4 \times 20)$

PAD-Gebühr

$(0,06 \mathrm{DM} / \mathrm{Min} \times 6 \times 4 \times 20)$

Zuschlag für Verbindungs-

gebühr

$(0,05 \mathrm{DM} \times 20)$

Zeitgebühr bei GVV

$(0,01 \times 6 \times 4 \times 20)$

Volumengebühr

$\left(\frac{2000}{64} \times 1,2 \times 6 \times 20 \times\right.$

$0,033 \mathrm{DM})$

$14,85 \mathrm{DM}$

$27,60 \mathrm{DM}{ }^{*} 138,-\mathrm{DM}$

$15,-\mathrm{DM}$

24,- DM

$28,80 \mathrm{DM}$

6,- DM

$4,80 \mathrm{DM}$
Belegungsgebühr TELE-

BOX

$(6 \times 4 \times 20 \times 0,30 \mathrm{DM}) \quad 144,-\mathrm{DM}$

Adressiergebühr

$(6 \times 20 \times 0,10 \mathrm{DM})$

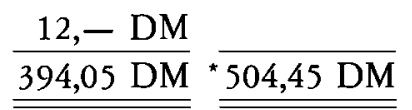

* bei künftig 0,23 DM je $50 \mathrm{sec}$

Übertragungszeit $5 \times 6 \times 20 \times 0,23 \mathrm{DM}$

Realisierung im DATEX-P-Netz:

1. Monatliche Gebübren:

1.1 DATEX-P10 Hauptanschluß 2400 $\mathrm{bit} / \mathrm{sec}$

$250,-\mathrm{DM}$

2. Verkebrs- (Mengen) Gebübren (Vg):

2.1 Jede Transaktion ca. 2 DIN A4-Seiten Zeicheninformation

Zeichen (Byte)

Gebübrenermittlung in Segmenten:

1 Segment max. 64 Bitgruppen zu je 8 Bit (1 Zeichen) monatliche Gebühren für die ersten 200000 Segmente:

$0,0033 \mathrm{DM} /$ Segment

$\mathrm{Vg}=\frac{2000}{64} \times 1,2 \times 6 \times 20 \times 0,0033 \mathrm{DM}$

$\mathrm{Vg}=14,85 \mathrm{DM}$

Zuschlagsgebühr je bereitgestellte Verbindung: 0,05 $\mathrm{DM} /$ Verb.

Zeitgebühr je gewährter virtueller Verbindung: 0,01 $\mathrm{DM} /$ Minute

$\mathrm{Vg}=6 \times 20 \times 0,05 \mathrm{DM}+6 \times 4 \times 20 \times 0,01 \mathrm{DM}$

$\mathrm{Vg}=10,80 \mathrm{DM}$

3. Monatliche Gesamtgebübren:

$250,-\mathrm{DM}$

$14,85 \mathrm{DM}$

$10,80 \mathrm{DM}$

$275,65 \mathrm{DM}$

\section{Realisierung im Btx-Dienst}

(Voraussetzung: Anschluß des externen Rechners an Btx)

Monatliche Gebübren:

1. Fernsprechhauptanschluß

2. Btx-Grundgebühr

$27,-$ DM

$8,-\mathrm{DM}$

\section{Verkehrsgebühren}

Es fallen Gebühreneinheiten von $0,23 \mathrm{DM}$ je 8 Minuten Ủbertragungszeit ein.

$\mathrm{Vg}=6 \times 20 \times 0,23 \mathrm{DM}$

$\mathrm{Vg}=27,60 \mathrm{DM}$ 


\begin{tabular}{|ll} 
3. Monatliche Gesamtgebübren: & $35,-\mathrm{DM}$ \\
& $27,60 \mathrm{DM}$ \\
\hline $62,60 \mathrm{DM}$ \\
\hline
\end{tabular}

Zusammenfassung des Gebührenvergleichs

Übertragung in Monatliche Gebühren

Fernsprechnetz mit Modem 1200-01

$595,75 \mathrm{DM}$

Datex-P-Netz

275,65 DM

Telebox

$394,05 \mathrm{DM}$

Deutsche Mailbox

$369,45 \mathrm{DM}$

Alexis

Btx

$376,45 \mathrm{DM}$

$62,60 \mathrm{DM}$

\section{Btx als kostengünstiges Zugriffsmedium}

Dieser Gebührenvergleich zeigt eindeutig, daß Bildschirmtext das mit Abstand preisgünstigste Medium für den Online-Datenbankzugriff ist. Für den gelegentlichen Nutzer von Datenbanken (das sind ca. 20000 Rechtsanwälte) gibt es hier keine große Kostenschwelle.
Ursache dafür ist vor allem ein Leistungsmerkmal: Btx ermöglicht eine kostengünstige Daten-Fernübertragung (DFÜ) kleinerer Datenmengen via Telefonleitung. Die DFU் kleinerer Datenmengen ist gleichzeitig ein Charakeristikum des Datenbank-Zugriffs für eine gelegentliche Datenbank-Nutzung.

Betrachtet man zu den günstigen Übertragungskosten die geringen Hardware-Kosten,

- ein Bildschirmtext-Arbeitsplatz, der mit einer alphanummerischen Tastatur ausgerüstet ist und damit die Ausnutzung der vollen Funktionalität normaler Text-Datenbanken ermöglicht, kostet ca. 1500,- DM,

- Datenbank-Terminals die von der Post und verschiedenen Herstellern unter der Bezeichnung Multitels angeboten werden, kosten je nach Komfort, monatliche Leasing-Raten zwischen $48,-\mathrm{u}$. $120,-\mathrm{DM}$,

dann ist es für den Eingeweihten unverständlich, daß noch keine Datenbank mit relevantem Inhalt für den juristischen Praktiker über Bildschirmtext angeboten wird. Da ist man uns in Frankreich einige Nasenlängen voraus. 